\title{
A provision-aware fair bandwidth distribution marker algorithm for DiffServ networks
}

\begin{abstract}
The rise in demand for real-time applications on the Internet necessitates Quality of Service (QoS). Differentiated Services (DiffServ) is one of the technologies used currently to provide QoS and service differentiation. It is simple and scalable. It provides service differentiation to aggregates, mainly through the Assured Forwarding (AF) per-hop behaviour. Previous work on fair sharing of network bandwidth did not adequately address the Under-Provisioned Network (UPN) condition. In this paper, we propose a new three-colour marker, named paItswTCM (provision-aware Improved TSW based Three-Colour Marker). We compare our new algorithm with both time-sliding window markers and token-bucket-based markers using simulations. Results show that our new provision-aware marker outperforms these previous algorithms not only in the UPN condition but also for low to medium network provision levels. We conclude that to achieve proportional sharing of bandwidth, no packet type should be injected at the expense of others. (C) 2010 Elsevier Ltd. All rights reserved.
\end{abstract}

Keyword: Differentiated services, Fairness 